Durante la sesión solemne estatutaria de la Academia Colombiana de Ciencias Exactas, Físicas y Naturales, llevada a cabo el 16 de agosto de 2017 en la Academia de la Lengua, se realizó la exaltación de Don Alberto Ospina Taborda como miembro honorario de la Academia y se reconoció a Don Juan Mayr Maldonado como amigo de la Academia.

Reproducimos las palabras de agradecimiento de los dos homenajeados

\section{Palabras de agradecimiento a la Academia Colombiana de Ciencias Exactas Físicas y Naturales}

Alberto Ospina Taborda*

\section{Agradecimiento e inspiración}

En diversas ocasiones he tenido el privilegio de disfrutar la cercanía, el calor y la amistad de los miembros de la Academia Colombiana de Ciencias Exactas, Físicas y Naturales. Pero es en la tarde de hoy cuando se da mi más sentido encuentro con ellos. Este será un acontecimiento para mantener por siempre en mi memoria; lo llevaré en el recuerdo y en el corazón el resto de mis días. La bendición divina y la generosidad de unos amigos de esta Academia me han dado el privilegio de acogerme hoy como uno de sus miembros honorarios. Al recibir este honor y agradecerlo, con humildad y con respeto, me complace compartirlo con mi familia, con mis amigos y con todas las distinguidas personas que aquí nos acompañan.

Siempre he admirado el alto nivel científico de las ilustradas personas que constituyen la Academia de Ciencias, así como su tenacidad y entusiasmo en el cumplimiento de su misión; mi ambición de siempre ha sido apoyar sus esfuerzos y contribuir al logro de sus aspiraciones en pro del avance de la ciencia en Colombia; nunca pensé en alcanzar el honor de sentarme a su lado. Es quizás por ello que me faltan palabras dignas del momento, para agradecer tan honrosa distinción.

Considero este día y este acto un punto de llegada; la culminación de un ascenso en la trayectoria de la vida profesional que comencé como soldado y marinero. Permítanme, entonces, evocar una frase en la oración de gratitud con la cual comenzábamos todos los días las jornadas militares; con ella afirmábamos nuestro amor por la tierra en que nacimos, por nuestros padres y nuestros hijos, por nuestros colegas, amigos y vecinos, por nuestros prójimos.

Con ella nos formamos, más que como militares, como ciudadanos; dice así el comienzo de la oración patria: “¡Colombia, patria mía, te llevo con amor en mi corazón!”. Esta fue, entonces, la oración inspiradora de mis sueños, mis actos y mis propósitos de vida.

\section{Construir la nación que soñamos}

Puede ser, igualmente, la fuerza orientadora que nos convoque a todos para construir la nación que soñamos, un país cada vez más próspero, una sociedad más justa, más feliz y en paz. Podremos alcanzar prosperidad si entendemos y abrazamos el progreso técnico, hijo del conocimiento y de la ciencia; podremos vivir en una sociedad más justa si logramos ponerle un límite al egoísmo individual en favor de la igualdad de oportunidades para todos; podremos vivir en paz si acogemos el perdón, si respetamos el derecho ajeno y las instituciones que nos rigen.

Apreciados amigos, sin tiempo hoy para recorrer esos tres caminos, los invito a unas breves reflexiones sobre el primero: el objetivo estratégico de lograr prosperidad para Colombia. Y a que, por esa ruta de reflexión constructiva, ayudemos a otros compatriotas más idóneos (del gobierno, de la academia y de la sociedad) en su empeño por el logro de los otros dos propósitos, la justicia social y la paz; pues solo con la sinergia de nuestros esfuerzos concertados podremos alcanzar, de la manera más eficaz, esos tres objetivos propuestos.

\section{Prosperidad como objetivo estratégico: innovación y progreso técnico}

Primera reflexión: ¿Recuerdan la locomotora de la innovación, esa feliz y sabia metáfora en pos de la prosperidad que nos llenó de ilusiones y de júbilo, a quienes teníamos puestas las esperanzas en el progreso técnico y el desarrollo de Colombia? ¿qué sucedió? ¿le faltó combustible al fallarle sus fuentes de financiación, el presupuesto nacional, el 10\% de las regalías? ¿le faltaron los rieles de una política pública y una voluntad genuina por quienes tenían responsabilidad y poder de decisión? ¿se enredó en la maraña de intereses políticos y trabas legales? ¿se erró el concepto de lo que es innovación en algún laberinto del Estado?

Para comenzar a responder estas preguntas era claro que primero había que armar la locomotora, o sea crear la capacidad de innovar. Sabemos que todavía se hacen esfuerzos para que esta máquina de la innovación arranque: nunca es tarde para encontrar y corregir los problemas que no le han permitido avanzar, o para contribuir a darle nuevo impulso, algo más difícil hoy y mañana, con los nuevos recortes presupuestales al sector de ciencia y tecnología.

Sea lo primero tener claridad sobre el concepto de innovación y entender su relación con el desarrollo de la ciencia y la tecnología. En su sentido más preciso, se tiene innovación cuando hay la capacidad de convertir el conocimiento 
en algo útil a la sociedad, cuando se tiene la habilidad de transformar ideas en nuevos procedimientos, nuevos productos y servicios para dar solución a los problemas de la comunidad y del país. Ello requiere, inexorablemente, algún nivel de conocimiento científico (ojalá el más alto), con sus aplicaciones tecnológicas, y de financiamiento del sector. Esta reflexión nos conduce, sin lugar a dudas, a la necesidad de unir nuestros esfuerzos para alcanzar ese nivel, con las personas y entidades que han tenido interés en la prosperidad del país... aunque buscada por ese camino hoy más que antes sin combustible y sin rieles.

Segunda reflexión: Se habla, a veces con patriótico entusiasmo, del desarrollo sostenible, del progreso técnico, de la inversión, del proceso de industrialización, del avance económico y social, como elementos necesarios para la ulterior prosperidad de los pueblos. Sin embargo, al mirar la situación actual en el caso colombiano, ¿hay evidencia de que se hayan definido e implementado políticas públicas dirigidas a lograr estos requisitos previos?

Hace más de cincuenta años, en los albores de Colciencias, cuando Colombia gozaba de una situación económica comparable o superior a la de muchas naciones de América y del sureste asiático (como Costa Rica, Perú, Chile, Corea, Indonesia, Singapur), estos países apenas empezaban a definir sus políticas públicas en educación, ciencia, tecnología e innovación, y comenzaba su ascenso en el camino de su desarrollo económico; Colombia superaba a casi todos ellos en grado de industrialización y en el monto de su producto interno bruto. Hoy algunos de esos países han alcanzado sus metas, sus niveles envidiables de bienestar; o están en pleno desarrollo económico y social.

Nos preguntamos, entonces: ¿qué hicieron sus dirigentes para lograr sus metas?, ¿qué no hicieron los nuestros, para que hoy la situación sea tan diferente y Colombia esté tan rezagada respecto a ellos, en lo concerniente a educación, ciencia, tecnología e innovación? ¿O en la prosperidad que nos podrían brindar el progreso técnico, el desarrollo económico y la equidad social? Es indudable que ellos diseñaron un proyecto integral de nación, en el cual las políticas públicas en educación, ciencia y tecnología fueron factores clave para realizarlo con eficacia; y, lo más importante, que las implementaron con vigor y decisión, y las mantuvieron en el tiempo.

\section{Una manera mejor: el poder del conocimiento}

Tercera reflexión, para concluir: en nuestra ansia de progreso y bienestar colectivo, hemos considerado el desarrollo económico, la equidad social y la paz, metas que aún no hemos alcanzado. Quizás haya una forma más eficaz para procurar la prosperidad y la felicidad de un pueblo; cuando nos lo proponemos, siempre encontraremos una manera mejor de ver y hacer las cosas. Intentémoslo. Los invito a reconocer y a poner nuestra esperanza en el poder del conocimiento y en las potencialidades que tiene Colombia para alcanzar su desarrollo sostenible: la capacidad e inteligencia de su gente, su institucionalidad y sus recursos naturales.

Sobre lo primero, el poder del conocimiento basta tan solo mencionar algo que enseñaba Robert Solov (1962), profesor de MIT y premio Nobel de economía: el conocimiento técnico es el que se pone en los bienes y servicios que producimos, vendemos o compramos; es el factor predominante de la producción, por encima de los tradicionales de la mano de obra, el capital financiero o los recursos de la Tierra. Y Thomas Steward (1997), otro economista connotado, en su obra sobre capital intelectual y siguiendo a Solov, remataba años más tarde con esta potente sentencia: "La información y el conocimiento son las armas termonucleares competitivas de nuestro tiempo"; y agregaba: "El conocimiento es más valioso y más potente que los recursos naturales, las grandes fábricas o gordas cuentas bancarias”.

Utilicemos entonces las armas de la información y el conocimiento para sacar provecho, con función social, de las potencialidades de Colombia: las mentes de su gran capital intelectual, las instituciones más eficientes, los elementos básicos de sus recursos naturales; o sean: las fuentes hídricas y las de energía, los minerales; su rica diversidad biológica, las especies vegetales útiles ya descubiertas, que son fuentes de medicinas y alimentos, bases de una economía competitiva. Aprovechemos y apropiemos el conocimiento científico y tecnológico que ya existe en el mundo para transformar esos recursos potenciales en riqueza nacional, prosperidad y desarrollo.

\section{Tarea}

Con esas herramientas, trabajemos un proyecto integrado de país que nos permita, además de vivir en paz con las necesidades básicas satisfechas, el crecimiento económico y la prosperidad como nación.

Los dejo con esas reflexiones. Quizás nos podamos encontrar en otra ocasión para discutir las conclusiones a las que ellas den lugar. Por ahora solo me queda reiterar mis sentidos agradecimientos por este acto que me honra, en el cual todos han contribuido; y compartir con ustedes la emoción de pertenecer a esta Academia, representada por una comunidad tan brillante, tan importante y tan capaz para lograr el progreso y el engrandecimiento de Colombia.

Vea Información suplementaria en: https://www.raccefyn. co/index.php/raccefyn/article/downloadSuppFile/530/2213

\section{Referencias}

Solov, R. Technical progress, capital formation and economic growth. The American Economic Review. 1962, 52 (2): 76-86.

Stewart, T A. Intelectual Capital. The new wealth of organizations (New York: Doubleday, 1997), p. ix. 


\section{Reflexiones sobre mi país}

Juan Mayr Maldonado*

Es para mí un gran honor recibir la distinción de amigo de la Academia, un reconocimiento que me lleva a recordar algunos pasajes de mi vida en los cuales tuve la fortuna de relacionarme con distinguidos académicos, que de una u otra forma han guiado mis pasos a lo largo de la vida.

Esa historia se remonta a la década de los 70, momento en el cual me inicie como editor y fotógrafo en las regiones de San Agustín y Tierradentro. Allí conocí a Luis Duque Gómez y a Julio Cesar Cubillos, con quienes sostuve numerosas charlas que despertaron mi interés por el misterioso mundo de la arqueología, un mundo que buscaba interpretar el pasado y la historia de las culturas de nuestro país. Al igual que el alto Magdalena, Tierradentro, otra región de geografía fantástica, me introdujo al mundo indígena de los paeces. Con estas semillas en mi mente me dedique a visitar y fotografiar las regiones más remotas de Colombia y a publicar libros sobre culturas prehispánicas, hasta que un buen día la prensa nacional anunció el hallazgo de una de las grandes ciudades Tayrona en las espesas selvas de la Sierra Nevada. Se trataba según los testimonios de los kogi, de Teyuna, uno de los sitios sagrados perteneciente a sus antepasados y hoy ampliamente conocida como Ciudad Perdida.

Gracias a Alvaro Soto, quien en ese momento se desempeñaba como director del Instituto Colombiano de Antropología y a su vez como director del Departamento de Antropología de la Universidad de los Andes, tuve la oportunidad de visitar y hacer las primeras fotografías de ese espectacular asentamiento precolombino que aún se encontraba cubierto por la selva. Ese día, que nunca olvidaré, nacía simultáneamente mi primer hijo, un día que cambiaría mi vida para siempre.

Desde ese momento inicié múltiples viajes a diferentes lugares en la Sierra Nevada, en especial al territorio de los kogi, y un par de años más tarde me integré al equipo interdisciplinario que trabajaba en el proyecto arqueológico de la Ciudad Perdida. Allí conocí y trabajé junto a biólogos, ingenieros, economistas, arquitectos, antropólogos, arqueólogos, médicos, en fin, un grupo de personas con distintas formaciones, que nos veíamos favorecidos por las visitas de importantes científicos nacionales y extranjeros. Entre ellos recuerdo la visita de Thomas van der Hammen quien, junto con un equipo de investigadores, realizó un transecto que dio a conocer la dinámica de los ecosistemas de la cuenca alta del rio Buritaca y con quien posteriormente me uniría una especial amistad.

Pero no sería solamente el contacto con científicos el que motivaba mi creciente interés por los conocimientos de las diferentes disciplinas. Un inesperado viaje a lo más profundo del territorio kogi, despertó en mí una inusitada curiosidad por entender un mundo tan diferente de aquel en el cual había nacido y me había criado. Ya son más de cuarenta años desde el momento en que se inició ese primer contacto que aún hoy se mantiene y se hace cada día más fuerte.

La grave problemática que para ese momento aquejaba a la Sierra Nevada, colonización, guaquería, deforestación, narcotráfico, mafias, y un poco más tarde las guerrillas y los paramilitares, eran una grave amenaza para la sobrevivencia de las culturas indígenas por la creciente violencia y la pérdida de su territorio tradicional. El Estado era en ese momento el gran ausente.

Tal situación nos llevó en ese entonces a un grupo de ciudadanos entre los que se contaban destacados científicos a crear, con el apoyo del presidente Belisario Betancur, la Fundación Pro-Sierra Nevada de Santa Marta de la cual fui su director por espacio de 13 años. Encabezados por Gerardo Reichel-Dolmatoff y su señora Alicia, fue posible crear el Comité Científico de la Fundación. Algunos de sus miembros eran Richard Evans Schultes, Jorge Hernández Camacho, Julio Carrizosa Umaña, José Francisco Socarrás, el premio Nobel Carleton Gaidesek, el famoso ornitólogo francés Jean Dorst, el geógrafo Olivier Dollfus y el recordado y querido director del Jardín Botánico de Bogotá y luego Presidente de la Academia de Ciencias Exactas, Físicas y Naturales, Luis Eduardo Mora Osejo. Los consejos y orientaciones del Comité Científico siempre fueron la guía para las acciones que emprendió la Fundación. A sus orientaciones se sumaron las de otros saberes, en especial las de los Mamas Mayores de los Kaggaba o kogis, con quienes se sembró la semilla para la creación de la Fundación y para el trabajo de protección del territorio tradicional, de sus ecosistemas y del conocimiento ancestral.

No me cabe duda que la visión integral y los resultados obtenidos por la Fundación durante sus primeros años en su trabajo con las comunidades indígenas y campesinas, los empresarios y diversos sectores gubernamentales, dieron como resultado un modelo innovador para aquella época que llamó la atención del mundo internacional y de las fundaciones de medio ambiente americanas y europeas las cuales, para ese momento, trabajaban por el medio ambiente bajo un patrón absolutamente proteccionista. La aproximación interdisciplinaria, con especial énfasis en lo social y lo cultural, y la concepción territorial de unidad geográfica y eco-región estratégica por encima de las divisiones político-administrativas - tres departamentos, diez municipios, resguardos indígenas y Parques Nacionales que se sobreponían los unos a otros-, despertó el interés de la Unión Mundial de Conservación de la Naturaleza. Las discusiones con el mundo científico en la UICN en Suiza y en numerosos eventos internacionales resultaron muy enriquecedoras y la Fundación pudo participar activamente en la construcción de la Estrategia Mundial para la Conservación de la Naturaleza, liderada por la UICN y otras importantes entidades internacionales en preparación para la Cumbre de Rio en 1992. 
Algunos de ustedes quizá recuerden que durante mi gestión como Ministro de medio ambiente entre 1998 y 2002, la política nacional ambiental llevó como tituló: El Proyecto Colectivo Ambiental, cuyo eje transversal era el agua. Así, junto a un gran equipo de profesionales y científicos adscritos al Sistema Nacional Ambiental, buscamos plasmar en las políticas públicas muchas de las experiencias y aprendizajes alcanzados en la Sierra Nevada. Allí, mediante un proceso de construcción colectiva con las comunidades y los diferentes sectores que dependen de esa compleja eco-región que se asemeja a una gran fábrica de agua, nos propusimos llegar mediante el diálogo y una amplia participación a acuerdos para diseñar un futuro común, tomando en cuenta las diferentes visiones y los distintos intereses. La información histórica e institucional, el conocimiento tanto local como científico, así como el apoyo académico de la Universidad de los Andes, fueron eje central para una participación informada. De esta manera las experiencias acumuladas en la Sierra Nevada fueron transferidas a otros territorios, todos ellos tan complejos como la misma Sierra, pero con otras características históricas, ecológicas, sociales, culturales, económicas y culturales.

También recuerdo para esa época un evento que hoy sigue siendo de gran actualidad. A mi despacho llegó una gran controversia entre el Distrito Capital y la CAR quienes no pudieron concertar algunos aspectos del POT, entre ellos las visiones contrapuestas sobre la expansión urbana en el borde norte de Bogotá.

Pues bien, el ministerio, que actuaba como juez en tan difícil controversia, en su afán por tomar la más correcta y acertada decisión, incorporó las experiencias aprendidas y estableció un panel de expertos del más alto nivel en el cual estaban figuras de la talla de Rogelio Salmona, Thomas van der Hammen, Julio Carrizosa, Manuel Rodríguez, Germán Samper y otros distinguidos profesionales, quienes con el apoyo de la Universidad Nacional como Secretaria Técnica, recomendaron crear la Reserva Forestal como garantía para mantener la estructura ecológica principal de la Sabana de Bogotá, proteger los acuíferos, generar una conectividad entre los cerros orientales, el cerro de la Conejera y el rio Bogotá, pero también para evitar la conurbación entre Chía y la Capital. Esta recomendación fue acogida por el ministerio y así nació la Reserva del Norte. Hoy, 17 años después el tema sigue siendo un tema candente y muchos intereses particulares buscan a toda costa evitar que la reserva, bautizada posteriormente con el nombre de Thomas van der Hammen en honor a sus grandes contribuciones científicas, pueda configurarse.

El contexto en que se desarrolló El Proyecto Colectivo Ambiental estaba enmarcado por tiempos de gran optimismo, más de 10.000.000 de personas habían votado por un mandato para la paz, lo que condujo a establecer un proceso de negociación con las FARC que, como todos nosotros conocemos, se vio frustrado. Fue también la época de las grandes negociaciones internacionales de medio ambiente en desarrollo de las Convenciones acordadas en la Cumbre de Rio de Janeiro, una de ellas el Protocolo de Bioseguridad, primer desarrollo legal de la Convención de Diversidad Biológica, un Protocolo de enorme importancia puesto que regulaba el movimiento transfronterizo de Organismos Vivos Modificados Genéticamente. Con el apoyo de diferentes sectores, grupos de expertos y científicos, y la voluntad política de más de 180 países, pude llevar a buen término esa negociación como presidente de la misma. Como muchas de las cosas que pasan en nuestro país, el Proyecto Colectivo Ambiental fue borrado de un plumazo una vez asumió el siguiente gobierno.

Como verán, a lo largo de mi vida he tenido la fortuna de contar con el consejo y apoyo de diferentes personalidades del mundo científico y académico, lo que me permitió más recientemente y por espacio de cinco años, como embajador de mi país en Alemania, concentrar todos los esfuerzos en conocer en profundidad el modelo alemán de educación, investigación, ciencia, tecnología e innovación, con el fin de establecer sistemas de cooperación con nuestros centros de investigación y universidades que le permitieran a nuestros estudiantes, académicos y científicos estrechar las relaciones con las mejores Sociedades de Investigación y universidades alemanas, entre las que se destacan las Sociedades Max Planck, Leibniz, Fraunhofer y Helmholz, todas ellas hoy con importantes lazos de cooperación con las entidades colombianas.

¿Quiénes no nos hemos hecho alguna vez esta pregunta? ¿Cómo es que Alemania, un país del cual no quedó piedra sobre piedra después de la Segunda Guerra Mundial, un país que quedó dividido y estigmatizado, un país sin recursos naturales, logro en siete décadas constituirse en una de las cinco economías más dinámicas del mundo?

Una parte muy importante de la respuesta tiene que ver con un sistema vigoroso de investigación, ciencia y tecnología en el cual el gobierno federal y los gobiernos regionales, trabajan de la mano con los sectores empresariales y académicos, en conjunto con el mundo científico. Es así como las decisiones políticas y empresariales toman muy en serio y con una visión de largo plazo la investigación y la ciencia como motor de la economía, el desarrollo y la innovación.

Con todas estas experiencias de vida, hoy cuando recibo la distinción de Amigo de la Academia, quisiera compartir con ustedes, algunas reflexiones sobre el momento que atraviesa nuestra querida Colombia.

Luego de más de medio siglo de confrontación armada que nos ha dejado, según el registro único de víctimas un total de 8.472.000 víctimas y más de 220.000 personas muertas, de las cuales 40.787 han sido combatientes, el gobierno ha alcanzado un acuerdo negociado con la mayor guerrilla de nuestro país. Las cifran mencionadas son solo una parte de los horrores de la guerra. Solo entre 1990 y 
julio de 2017 se registraron 7.220.000 desplazados y hasta 2015 se registró un total de 106.000 desaparecidos de los cuales 22.227 son desapariciones presuntamente forzadas. Todos ellos compatriotas víctimas del conflicto interno y la violencia. A estas cifras podríamos agregar otras sobre el flagelo del secuestro, de las víctimas de minas antipersona, del reclutamiento de menores, de los delitos sexuales contra las mujeres como arma de guerra y múltiples violaciones a los Derechos Humanos.

Al conocerlas uno no puede más que horrorizarse y a la vez avergonzarse. Como colombianos no podemos desconocer lo que nos ha dejado el horror de la guerra, como tampoco los beneficios inmediatos desde el momento en que se decretó el cese al fuego con las FARC, beneficios que se han traducido en una reducción significativa de las víctimas del conflicto. Según CERAC el proceso de paz, en comparación con el acumulado histórico, previno la muerte de 1.553 guerrilleros y 556 miembros de la fuerza pública, pero también de integrantes de los grupos más vulnerables en nuestro país, hablo de campesinos e indígenas. Una sola vida salvada justifica lo acordado.

Colombia ha logrado dar un paso enorme con el acuerdo de paz al que se ha llegado con las FARC. No solo se han salvado cientos de vidas, sino que se abren nuevos espacios para fortalecer nuestra democracia al cambiar las armas por argumentos e ideas. Un acuerdo que permitirá avanzar hacia un mayor control y presencia estatal en el territorio nacional e integrar todas aquellas regiones marginadas y a su gente, al desarrollo del país a la vez que nos permitirá avanzar hacia una sociedad más participativa e incluyente, hacia una democracia en la que quepamos todos como lo demanda y requiere nuestro país.

Sin embargo, a pesar del respaldo unánime de la comunidad internacional al más alto nivel y de los enormes esfuerzos que ha implicado la negociación de paz, una negociación que ha reconocido y puesto a las víctimas en el centro de la atención, los acuerdos aún son frágiles y corren el riesgo de que su implementación quede a medio camino o que los acuerdos sean hechos trizas, como algún conocido político lo ha anunciado. La incorporación de los exguerrilleros a la sociedad todavía está por verse y los más recientes asesinatos de líderes sociales y políticos, al igual que de excombatientes y sus familiares generan enormes dudas e incrementan la desconfianza para que otros grupos, como es el caso del ELN, se atrevan a dar el paso para avanzar hacia una paz total.

Si bien el acuerdo con las FARC es un paso gigantesco en la dirección correcta y nos plantea aún grandes desafíos para la construcción de una paz sostenible y duradera, no podríamos dejar de lado, en una mirada de largo plazo, otros muchos desafíos que nuestro país continúa teniendo por delante y en los cuales las Academias y las universidades están llamadas a convertirse en actores de primera línea.

A pesar de los avances en la reducción de la pobreza, seguimos siendo uno de los países más inequitativos de la región y del mundo; nuestro vecindario está que arde; los grupos delincuenciales y el narcotráfico están aún vivos; la corrupción ha llegado a límites insostenibles mientras el desprestigio de la clase política es enorme; la época de las vacas gordas es cosa del pasado y nuestra economía muestra síntomas de debilitamiento, además sigue estando basada en la extracción de recursos naturales en una gran proporción; también somos uno de los países más vulnerables al cambio climático y nuestras políticas de ordenamiento territorial son deficientes como también lo son nuestras políticas ambientales. Con estos pocos ejemplos podemos entender que aún hay mucho camino por recorrer para proyectarnos hacia un país más equitativo y que como dice mi buen amigo el padre Francisco de Roux, un país que nos permita a todos vivir como seres humanos.

No me cabe duda de que como colombianos nos estamos jugando el futuro en medio de una sociedad polarizada y fragmentada como nunca, lo cual imposibilita tener una visión compartida de futuro. La desconfianza, el odio y el pesimismo carcomen a la sociedad, la crisis de valores salta a la vista y todo esto ad portas de una campaña electoral.

Es precisamente en estos momentos de gran dificultad y enormes retos que Colombia necesita la voz de sus mejores cerebros para construir una nueva narrativa de país que convoque a todos los ciudadanos. Dejar nuestro futuro común en manos de otros podría llegar a ser de gran riesgo. Una narrativa donde prime una visión ética para la construcción de un nuevo país en el marco de un desarrollo sostenible. Hoy más que nunca se requiere del noble compromiso de las mentes más ilustres con que cuenta nuestra sociedad con el fin de que eleven su voz y orienten, con su conocimiento y buen ejemplo, a la comunidad y a sus dirigentes para que mediante un esfuerzo común asumamos los desafíos del futuro, que como ya hemos visto no son de poca monta.

Ese es el llamado que yo, como amigo de la Academia, quisiera hacerles hoy aquí. Estoy seguro de que una gran alianza de las Academias reunidas en el Colegio Máximo de las Academias, junto a una alianza de las universidades del país es una voz que ningún colombiano puede ignorar.

Vea Información suplementaria en: https://www.raccefyn. co/index.php/raccefyn/article/downloadSuppFile/530/2213 